\title{
Music Performance
}

\section{William Forde Thompson'1, Simone Dalla Bella², and Peter E. Keller ${ }^{3}$}

\author{
${ }^{1}$ University of Toronto at Mississauga, Toronto, Canada \\ 2 Department of Cognitive Psychology, University of Finance and Management, Warsaw, Poland \\ ${ }^{3}$ Department of Psychology, Max Planck Institute for Human Cognitive and Brain Sciences, Leipzig, Germany
}

The ability to perform music is widespread in the general population (e.g. singing), but few individuals develop a high level of musical proficiency. Skilled musicianship typically requires decades of regular practice, estimated at 10,000 hours (Ericsson, Krampe, \& Tesch-Römer, 1996; Howe, Davidson, \& Sloboda, 1998). Through this prolonged engagement with music, performers acquire outstanding motor skills and an enormous body of knowledge about music. Over the past two decades, this knowledge and skill has been the subject of intense scientific investigation. In this special issue, leading researchers of performance review their recent and ongoing investigations in four broad areas of focus: applied cognitive psychology, analysis-by-synthesis, movement control, and neuroscience.

\section{Applied cognitive psychology}

Skilled performers employ a large number of expressive strategies that often take decades to master effectively. Whereas a musical score provides detailed information about pitch and rhythmic structure, it provides little guidance to performers about the expressive nuances that are needed to bring music to life, including the expressive use of timing (e.g., rubato, accelerandi), intensity (e.g., accents, crescendi), intonation, and articulation. Successful performance requires exceptional motor skills and an extensive knowledge of musical structure and performance traditions.

With so much knowledge and skill to manage, and with the pressure of having to perform in front of an audience, it is not surprising that performers often become anxious. Indeed, music performance anxiety (MPA) is a serious problem that has prevented many excellent musicians from pursuing a career in music. Kenny and Osborne (this volume) summarize their work on MPA, focusing on young musicians.
They propose that performance anxiety often begins early in musical development, and many characteristics of the disorder remain into adulthood. As Kenny and Osborne explain, fear of failure often becomes a source of distraction that leads to poor performance, thereby reinforcing the association between performance and anxiety. The authors outline two inventories to assess a musician's susceptibility to MPA. The Kenny Music Performance Anxiety Inventory (K-MPAI) (Kenny et al., 2004) was developed for adults, whereas the Music Performance Anxiety Inventory for Adolescents (MPAI-A) (Osborne \& Kenny, 2005) was developed for younger musicians. Both scales show much promise as a predictor of MPA and as outcome measures following treatment for MPA.

Chaffin and Logan (this volume) point out that while concert music is usually performed from memory and hence must be practiced until its production can be carried out automatically, the performance should appear spontaneous. The research reviewed by Chaffin and Logan suggests that the solution to this paradox lies in how musicians prepare for their performances. Effective practice sessions are typically aimed at the formation of a mental map of a piece, which is acquired by attending to landmarks in the hierarchical structure of the music. These landmarks serve as cues that help the musician to monitor the automatic aspects of playing while adapting spontaneously to the unique conditions that accompany each performance.

\section{Analysis-by-synthesis}

If expressive actions act upon musical structure in a systematic way, then it should be possible to define

Inquiries or requests for reprints should be sent to: William Forde Thompson, University of Toronto at Mississauga, 3014 CCT Building, 3359 Mississauga Road North, Mississauga, Ontario, L5L 1C6 Canada, b.thompson@utoronto.ca 
these actions in the form of a rule system, much like a "grammar" of performance. Moreover, it should be possible to implement this rule system using computer-based tools to generate "automatic" performances. By listening to the results of such an exercise (or conducting perceptual / evaluative experiments on music that has been shaped by the rules), it should be possible to further refine the rule system. This approach to the study of music performance has been pioneered by the KTH (Kungliga Tekniska Högskolan - Royal Institute of Technology, Stockholm) over the last three decades.

Sundberg (this volume) reviews research on the synthesis of sung performance. His initial attempts to synthesize the singing voice were carried out in 1970's (e.g., the KTH Music and Singing Synthesis Equipment, or MUSSE; RULSYS). Stimuli generated with MUSSE were of excellent vocal quality, but when placed into a musical context the performances sounded unmusical. To address this challenge, Sundberg devised a set of rules for singing synthesis that make up the RULSYS method (e.g., Duration of consonants rule; Timing of pitch change rule). Sundberg describes a number of applications of these systems, ranging from understanding the "singer's formant" (useful for vocal projection) to characterizing the qualities of an "ugly" singing voice. He concludes by arguing that analysisby-synthesis is a powerful tool for understanding the acoustical properties of the singing voice.

Friberg, Bresin and Sundberg (this volume) next describe the longstanding attempts by members of $\mathrm{KTH}$ to devise a rule system that captures the principles by which Western musicians perform classical, jazz, and popular music. The project began roughly 25 years ago when a renowned pedagogue and violin player (Lars Frydén) was asked a simple question: What do you teach your students? The answer, it seems, is more complicated than one might imagine: more than two and a half decades later the goal of articulating a core set of rules for the performance of Western tonal music is still in progress. Rules that initially seem self-evident turn out to be highly conditional, and combinations of rules have unexpected effects. Each year, new rules are introduced and tested both in isolation and in combination with existing rules. As such, an up-to-date review of the $\mathrm{KTH}$ rule system is both illuminating and fascinating. Prior to this special issue, the most recent overview of the rule system was published in 1995 as part of Anders Friberg's doctoral dissertation. Since then, various rules have been introduced, including rules for articulation, ensemble swing in jazz, and the mod- eling of neural motor noise. Improvements to existing rules have also been introduced.

\section{Movement control}

The ability of skilled musicians to coordinate hand and finger movements during performance is impressive and sometimes even mesmerizing. A skilled pianist has the potential to produce movements at rates that exceed visual reaction times (e.g., in the case of trills; Lashley, 1951), often playing up to 30 sequential notes per second over extended passages (Rumelhart \& Norman, 1982). Once the cognitive plans that guide musical performance have been established, activation of an appropriate plan triggers the motor programs that drive and control the movements that are required to create the sound (Gabrielsson, 1999; Palmer, 1997; Shaffer, 1985). A musician must continuously monitor his or her activity - and often the activity of other musicians - through sensory feedback in order to engage in error correction when the need arises (Pressing, 1999).

In the first contribution to this section, Repp (this volume) addresses the upper and lower rate limits within which individuals can synchronize simple movements (finger taps) with auditory and visual sequences. These rate limits constrain most musical activities involving two or more individuals, and hence they are relevant across diverse musical styles and cultures. The work that Repp describes reveals that synchronization thresholds (i.e., the fastest and slowest rates at which synchronization is possible) are determined not only by biomechanical limitations, but also by structural aspects of the pacing signal (e.g., auditory vs. visual modality, rhythmic complexity, and hierarchical organization). This leads Repp to conclude that rate limits in musical synchronization derive largely from constraints on the sensorimotor integration processes that link incoming perceptual information with motor commands.

In the next contribution, Pfordresher (this volume) carries the theme of sensorimotor integration into the realm of more complex movement sequences. He describes research using a paradigm in which auditory feed-back is altered during the performance of piano melodies. Although the removal of auditory feedback has negligible effects on piano performance, certain types of altered feedback are profoundly disruptive. After charting the relative disruption caused by different types of altered feedback (e.g., changes in pitch content vs. temporal misalignment), Pfordresher offers a theoretical account of how melodies and the 
movement sequences that produce them are corepresented in memory. This account is consistent with the view that processes underlying perception and action operate in a common representational domain (e.g., Hommel, Müsseler, Aschersleben, \& Prinz, 2001).

\section{Neuroscience of performance}

The performance of music likely engages a distributed network of brain regions involving motor planning, fine motor coordination, timing, and memory retrieval (Tramo, 2001). Hence, studying the neuronal underpinnings of music performance provides a unique opportunity to gain insight into the operation and coordination of several brain functions. Although relatively little is known about brain regions that are responsible for specific processes in skilled music performance, such as memory retrieval or motor planning, it has been confirmed that extensive musical practice significantly affects brain connectivity in musicians (Schlaug, 2001). For example, long years of practice in professional musicians are associated with enlarged cortical representations in the somatosensory and auditory regions of the brain (Pantev et al., 2001).

This section includes three contributions addressing the consequences of brain lesion or brain malfunctioning on music performance and reading, and the neuronal underpinnings of music expectancies. Hébert and Cuddy (this volume) present an extensive review of the neuropsychological literature on the effects of brain damage on music reading abilities. Music reading is essential to music performance (e.g., in the case of sightreading). Their review of existing research supports the idea that music reading is a complex ability that results from the operation of several subfunctions, and that these subfunctions are potentially dissociable following brain damage. Hébert and Cuddy provide evidence of a double dissociation between text and music reading and review finegrained dissociations within music-reading abilities. They raise the interesting possibility that music-specific developmental reading deficits mirror developmental dyslexia. In the end, the authors conclude that music reading, like music perception and performance generally, is not a monolithic ability but a modular system formed by several subsystems, some of which may be specialized for music processing.

The contribution by Jabusch and Altenmueller (this volume) is devoted to the origins and treatment of focal dystonia (i.e. musician's cramp). Focal dysto- nia is a loss of coordination or voluntary motor control emerging while a musician is playing an instrument, often leading to a halt in performers' musical career. Jabusch and Altenmueller review the epidemiology of focal dystonia, paying close attention to the risk factors that may lead to the impairment. Evidence bearing on the neuronal underpinnings of focal dystonia is reviewed, and suggests that the disorder is associated with deficits at the level of the basal ganglia, the sensory thalamus, and the cortex. Finally, the authors report the results of a study on the effectiveness of different treatment strategies of focal dystonia (e.g., pharmacological therapies and pedagogical training). They conclude that even though some patients continue to perform after engaging in therapy, the available therapies of focal dystonia are largely ineffective.

As Zanto, Snyder, and Large (this volume) note in the final contribution to the special issue, music performance is a behaviour in which "complex sequences of events are exquisitely structured in time" (p. 221). As such, processes of rhythmic expectancy must be engaged in order to achieve the high level of temporal precision and sequencing that is required for successful musical performance. Zanto et al. describe a growing body of work on the neuroelectric correlates of rhythmic expectancy. Such correlates have been found in event-related brain responses that occur when an event is omitted from a periodic auditory sequence. These induced responses, which are observed as transient activations in gamma band (20-60 Hz) EEG, precede the time point at which the omitted event should have occurred and are sensitive to structural variations in the sequence (such as accent pattern). Zanto et al. suggest that these activations may reflect the operation of an attentional mechanism that facilitates music perception and production by allowing the listener or performer to generate temporally precise expectancies.

The contributions that make up this special issue represent some of the major approaches to the examination of music performance, and indicate the relevance of this activity to several important areas within cognitive science. Performance encompasses a wide range of cognitive and motor skills, all of which are coordinated to an exquisite degree. As such, it represents a rich area for scientific study. A number of recent books on performance attest to the growing interest in the topic, including an important book edited by Richard Parncutt and Gary McPherson (2002) on the pedagogical implications 
of performance research, and a more recent book edited by Aaron Williamon (2004), which considers strategies and techniques for enhancing performance excellence (see review by $D$. Kenny, this issue). Performance excellence is valued across musical traditions because it contributes to effective musical communication. Listeners attend closely to the expressive qualities of music because they clarify and emphasize the emotional, stylistic, and structural dimensions of music. In some cases, we are simply amazed by the shear skill involved - a reaction most famously exemplified by audience responses to performers such as Franz Liszt, Antonio Vivaldi and Ignace Jan Paderewski. Highly skilled performers may represent a relatively small percentage of the population, but virtually all listeners are deeply moved by the results of their expertise. As the contributions of this special issue make clear, we are beginning to gain a clearer understanding of this complex and fascinating human behavior.

\section{References}

Chaffin, R., \& Logan, T. (2006). Practicing perfection: How concert soloists prepare for performance. Advances in Cognitive Psychology, 2, 113-130. A C P Ericsson, K. A., Krampe, R. Th., \& Tesch-Römer, C. (1993). The role of deliberate practice in the acquisition of expert performance, Psychological Review, 100, 363-406.

Friberg, A., Bresin, R., \& Sundberg, J. (2006). Overview of the KTH rule system for musical performance. Advances in Cognitive Psychology, 2, 145-161. A C P

Gabrielsson, A. (1999). The performance of music. In D. Deutsch (Ed.), The Psychology of Music (2nd ed., pp. 501-602). San Diego: Academic Press.

Hébert, S., \& Cuddy, L. L. (2006). Music-reading deficiencies and the brain. Advances in Cognitive Psychology, 2, 199-206. A C P

Hommel, B., Müsseler, J., Aschersleben, G., \& Prinz, W. (2001). The theory of event coding (TEC): A framework for perception and action. Behavioral \& Brain Sciences, 24, 849-937. $\overline{w w w}$

Howe, M. J. A., Davidson, J. W., \& Sloboda, J. A. (1998). Innate gifts and talents: Reality or myth? Behavioral \& Brain Sciences, 21, 399-407. | $\mid \mathrm{ww}$

Jabusch, H.-C., \& Altenmüller, E. (2006). Focal Dystonia in musicians: From phenomenology to therapy. Advances in Cognitive Psychology, 2, 207-221. A C P

Kenny, D. T., Davis, P. J., \& Oates, J. (2004). Music performance anxiety and occupational stress amongst opera chorus artists and their relationship with state and trait anxiety and perfectionism. Journal of Anxiety Disorders, 18, 757-777.

Kenny, D. T., \& Osborne, M. S. (2006). Music performance anxiety: New insights from young musicians. Advances in Cognitive Psychology, 2, 103-112. A C P Lashley K. (1951). The problem of serial order in behavior. In L.A. Jeffress (Ed.), Cerebral Mechanisms in Behavior: The Hixon Symposium (pp.11236). New York: Wiley.

Osborne, M. S., \& Kenny, D. T. (2005). Development and validation of a music performance anxiety inventory for gifted adolescent musicians. Journal of Anxiety Disorders, 19, 7, 725-751. Www

Palmer, C. (1997). Music performance. Annual Review of Psychology, 48, 115-138. Www

Pantev, C., Engelien, A., Candia, V., \& Elbert, T. (2001). Representational cortex in musicians. Plastic alteration in response to musical practice. Annals of The New York Academy of Sciences, 930, 300-314. |www

Parncutt, R. \& McPherson, G. (2002). The Science and Psychology of Music Performance: Creative Strategies for Teaching and Learning. New York: Oxford University Press.

Pfordresher, P. Q. (2006). Coordination of perception and action in music performance. Advances in Cognitive Psychology, 2, 183-198. A C P

Pressing, J. (1999). The referential dynamics of cognition and action. Psychological Review, 106, 714-747.

Repp, B. H., (2006). Rate Limits of Sensorimotor Synchronization. Advances in Cognitive Psychology, 2, 163-181. A C P

Rumelhart, D.E., \& Norman, D.A. (1982). Simulating a skilled typist: A study of skilled cognitive-motor performance. Cognitive Science, 6, 1-36.

Schlaug, G. (2001). The brain of musicians. A model of functional and structural adaptation. Annals of The New York Academy of Sciences, 930, 281-299. [www

Shaffer, L. H. (1985). Timing in action. In J. A. Michon \& J. L. Jackson (Eds.), Time, mind, and behavior (pp. 226-241). Berlin: Springer-Verlag.

Sundberg, J. (2006). The KTH Synthesis of Singing. Advances in Cognitive Psychology, 2, 131-143.

Tramo, M.J. (2001). Biology and music. Music of the hemispheres. Science, 291(5501), 54-6. |www

Williamon, A. (Ed.) (2004). Musical Excellence: Strategies and techniques to enhance performance. Oxford: Oxford University Press

Zanto, T. P., Snyder, J. S., \& Large, E. W. (2006). Neural correlates of rhythmic expectancy. Advances in Cognitive Psychology, 2, 221-231. A C P 